\title{
MEJORA GENÉTICA: LO QUE EL VIENTO NOS TRAERÁ
}

\author{
Rosario Sierra de Grado
}

Instituto Universitario de Gestión Forestal Sostenible. Dpto. Producción Vegetal y Recursos Forestales. Escuela Técnica superior de Ingenierías Agrarias (Palencia). Universidad de Valladolid. Avenida de Madrid 44. 34071-PALENCIA (España)

\section{Resumen}

Cada vez más, en la mejora genética forestal, los términos de la tradicional fórmula Fenotipo = Genotipo + Ambiente se entienden como multidimensionales. Las actuales herramientas genómicas posibilitan obtener una enorme información sobre los genotipos, pero para poder aprovecharla en la mejora, es necesario desarrollar mapas genotipo-fenotipo que permitan comprender mejor las relaciones entre ambos. Dado que los caracteres de interés son sumamente complejos (crecimiento, calidad de la madera, resistencia a patógenos, etc.), entender qué procesos funcionales subyacen bajo estos caracteres y cómo interaccionan con el ambiente son cuestiones clave para poder realizar una selección adecuada. Por otra parte, es necesario considerar fenotipos de forma integral, ya que la energía y recursos disponibles para realizar las funciones vitales (crecimiento, reproducción y mantenimiento) son limitados, por lo que la selección sesgada hacia una de esas funciones puede comprometer la optimización simultánea de las demás. En cuanto al ambiente, la longevidad de los árboles impone seleccionar considerando las posibles condiciones ambientales futuras en un contexto de cambio climático. Esta ponencia analizará este panorama multidimensional y argumentará la necesidad de integrar en la selección fenotípica la estabilidad mecánica y de incluir el viento entre los factores ambientales principales que explican el comportamiento de los genotipos. Para los árboles, seres de larga vida y gran tamaño, mantenerse en pie en un ambiente sometido a esfuerzos mecánicos variables, como las cargas debidas al viento, nieve, etc., no es una función trivial. Lo consiguen mediante mecanismos de control postural y desarrollando un buen sistema de anclaje (raíces), que pueden comprometer el crecimiento a través del reparto de biomasa aérea/radical. También repercuten en la forma del tronco. En estos procesos puede haber componentes con variabilidad genética y heredables. Las previsiones de cambio climático contemplan un notable incremento de daños por viento (derribos) en Europa en este siglo. Pero además, el régimen ordinario -no catastrófico- del viento tiene efectos notables ya documentados sobre el crecimiento, la arquitectura y la rectitud de los troncos, que lo sitúan como una variable ambiental explicativa de primer orden. Incluir estos aspectos en los programas de mejora genética puede incrementar la eficacia de los mismos en su objetivo final de obtención de madera en cantidad y calidad.

Palabras clave: Fenotipo, Genotipo, Variabilidad genética, Crecimiento, Madera, Ambiente 


\section{LA MEJORA GENÉTICA FORESTAL Y LOS CARACTERES OBJETIVO}

El objetivo general de los programas de mejora genética (PMG) de árboles puede formularse como desarrollar variedades mejoradas conforme a unos objetivos que se adapten bien a los sitios donde se van a plantar. Un ejemplo relevante, por su importancia económica y la extensión de las plantaciones realizadas, son los PMG de coníferas llevados a cabo en todo el mundo durante los últimos 50 años (ver la extensa revisión de MulLin et al., 2011). Estos programas han llegado a suministrar material forestal de reproducción mejorado para extensivas repoblaciones, y en algunos países hay programas que ya han desarrollado la tercera e incluso la cuarta generación.

Como ejemplo podemos citar Pinus pinaster en Las Landas, que cuenta con huertos semilleros y ensayos de progenies de tercera generación. Los sucesivos materiales generados por los PMG han abastecido de semilla a esta extensa región de cerca de un millón de hectáreas, incluidas las grandes reforestaciones que han seguido a desastres como la intensa helada de 1985, que destruyó 30.000 ha de pinar y el huracán de 1999 que derribó unos 100.000 árboles, reforestándose a continuación la superficie arrasada con semilla de huertos de segunda generación.

En los PMG de especies forestales, uno de los grandes retos es la reducción del ciclo de mejora. En muchas de las principales especies forestales, y en particular para los pinos, cada ciclo de mejora dura entre una y dos décadas (RESENDE et $a l$., 2012), en buena medida porque se requieren esos periodos para poder evaluar fenotípicamente y seleccionar con suficiente fiabilidad.

Estos programas se han centrado principalmente en tres tipos de caracteres: crecimiento, forma y resistencia a agentes bióticos y abióti$\cos$. En los últimos años ha comenzado a generalizarse un esfuerzo por incluir caracteres de calidad de madera y adaptación con vistas al cambio climático.

Todos ellos son caracteres complejos, es decir, implican la expresión coordinada de muchos genes y responden integradamente a diversas condiciones ambientales, que actúan a la vez y que van cambiando a lo largo de la vida del árbol.

\section{El modelo $\mathbf{F}=\mathbf{G}+\mathbf{A}$}

Los caracteres de mejora mencionados han sido considerados generalmente como cuantitativos y su tratamiento en los PMG clásicos ha sido bajo el modelo $F=G+A$, donde el valor fenotípico $\mathrm{F}$ de un individuo se expresa como la suma de su valor genético $\mathrm{G}$ y el efecto ambiental residual $A$, y en el que $G$ incluye los efectos genéticos aditivos, de dominancia y epistasis y A todos los efectos no genéticos (ZoBEL \& TALBERT, 1984). A este modelo se le suele añadir otro término de interacción GxA cuando se analizan varios genotipos en varios ambientes, que denota posibles diferencias en la plasticidad de los genotipos (CHAmBel et al., 2005).

El término fenotipo suele referirse a un carácter medible de un organismo, lo que muchas veces hace depender la definición del "fenotipo" de nuestra capacidad de medida. Así como el genotipo está circunscrito a la dotación genética, el fenotipo puede definirse en muy diferentes escalas de organización biológica. Además, la dependencia del fenotipo de su contexto ambiental (que va desde las condiciones climáticas a la presencia de otros genes en el genoma del individuo, así como de la fase del desarrollo del organismo o, incluso, del momento del día para algunos caracteres, pasando por modificaciones epigenéticas, interacción con otros organismos y un largo etcétera de condicionantes) hace que sus posibles variaciones sean muchas. Por tanto, no debemos olvidar que la ecuación $\mathrm{F}=\mathrm{G}+\mathrm{A}$ no deja de ser un modelo (y por tanto una simplificación), y bastante sencillo además, que permite un avance limitado en nuestra comprensión de las relaciones entre el fenotipo, el genotipo y el ambiente.

Tradicionalmente se ha utilizado la metáfora de la caja negra para referirse a las relaciones fenotipo-genotipo. El desconocimiento que visualiza esa imagen probablemente sea la base de la falta de eficiencia en los PMG en muchas ocasiones. Las preguntas sobre qué genes juegan un papel relevante en cada uno de estos caracteres, qué funciones desempeñan, cuáles son los responsables de la variación observada entre especies y poblaciones, qué procesos ligan los genotipos con los fenotipos y cómo se ven afectados por las condiciones ambientales son sin duda claves para avanzar en nuestro conocimiento y capacidad de manejo de la variabilidad genética. 


\section{El Genotipo (G)}

Hace pocos años, la capacidad de conocer el genotipo de los individuos evaluados era bastante reducida, pero la tecnología ha experimentado un desarrollo exponencial y su aplicación actual posibilita la obtención de gran cantidad de datos genéticos en poco tiempo y a un coste cada vez menor. Esta capacidad tecnológica ha conllevado el cambio de escala en los objetos de estudio: así, hemos pasado, en un lapso de pocos años, de los marcadores fenotípicos a los genéticos y de estos a los genómicos, así como de la genética de poblaciones a la genómica de paisajes.

La posibilidad de avanzar en la mejora genética de árboles que proporcionan las nuevas tecnologías es enorme, y hay grandes esperanzas depositadas en que de ellas derivarán incrementos importantes en la efectividad de la selección y de la reducción de los ciclos de mejora (GONZÁLEZMARTíNEZ et al., 2007; BusOv et al., 2008; GASPAR et al., 2009; MuLLIN et al., 2011, RESENDE et al., 2012). Los marcadores moleculares y las técnicas de genotipado de alto rendimiento pueden aplicarse al estudio de la diversidad genética, estudios de asociaciones, mapas genéticos, selección asistida por marcadores, identificación de genes candidatos para QTLs (quantitative trait loci), etc. Por otra parte la transformación genética de plantas, genética reversa, análisis de transcriptos y niveles de expresión génica están siendo útiles para desenmarañar la funcionalidad de los genes (SOMERS et al., 2009; OrAGUZIE et al., 2007; JANNSON et al., 2010).

Junto con el desarrollo de dichas tecnologías, el uso de especies modelo o de referencia (Arabidopsis thaliana, Oryza sativa, Populus trichocar$p a$, etc.) ha concentrado muchos esfuerzos de miles de investigadores en todo el mundo. La secuenciación de sus genomas y el estudio de las asociaciones entre genes y caracteres, muchas veces extrapolables a otras especies, está permitiendo un cada vez más rápido avance en la comprensión de la biología vegetal (ELLIS et al., 2010).

Y no menos importante para el avance científico que todo lo anterior, es el desarrollo de gigantescas bases de datos genómicos, fenotípicos y ambientales, la posibilidad universal de acceso a esa información vía internet y la potencia de cálculo estadístico de los ordenadores. Un ejemplo de ello, especialmente enfocado a especies forestales es CartograTree, que conecta datos ambientales, genómicos y fenotípicos sobre una base cartográfica geo-referenciada (VASQUEZ-GROss et al., 2013).

\section{El Ambiente (A)}

Las condiciones ambientales que influyen en las características de los árboles son múltiples (no sólo climáticas), actúan solapadamente, interaccionando entre sí y con el propio organismo. Por ejemplo, las características ambientales que afectan a la capacidad de un árbol para resistir al viento son resultado de una interacción compleja entre la velocidad del viento, la duración del vendaval, el tipo de suelo y su estado -agravándose el riesgo de fallo cuando está encharcado- y las características del rodal, incluidas sus condiciones de manejo (GARDINER, 2010).

La selección de genotipos en los PMG se realiza para su uso en un rango de ambientes determinados y basándose en ensayos establecidos en lugares y años concretos. Pero ¿hasta qué punto son extrapolables los resultados obtenidos a otros lugares o tiempos? ¿Cómo optimizar la selección para obtener las mejores ganancias si las condiciones ambientales cambian en el tiempo o en el espacio?.

Dada la longevidad de los árboles y los largos turnos de su aprovechamiento, la selección en los PMG no debe realizarse sin tener en cuenta las previsiones de condiciones ambientales futuras en un contexto de cambio climático. Existe un consenso en la comunidad científica sobre el impacto rápido y severo que el cambio climático está teniendo en zonas sensibles como el Sur de Europa (COST 4131/12, MÁTYÁs et al., 2009). También hay acuerdo en que el cambio climático es multifactorial y deben tenerse en cuenta tanto los cambios progresivos como los impactos de eventos extremos. Se presupone, así mismo, una importante interacción GxA en las respuestas de diferentes poblaciones a cambios climáticos (MÁTYÁs, 2000). Los criterios de selección en un panorama de ambiente cambiante deben incluir la capacidad de adaptación, y se deben definir las zonas de transferencia de semilla de manera acorde (CHAMBEL et al., 2005).

\section{El Fenotipo (F)}

Los fenotipos son finalmente los que integran la variabilidad genética y ambiental de todo el sistema o sistemas implicados. En el ámbito de las llamadas "ómicas" (genómica, transcriptómi- 
ca, proteómica, fenómica, etc.) se ha definido fenoma como "la representación fenotípica completa del individuo o la especie" (FrEIMER \& SABATTI, 2003), en paralelo a la definición de genoma como totalidad de la información genética que posee un organismo o una especie en particular. Sin embargo un fenoma completo, en sentido paralelo al de "genoma completo" es más difícil de definir, teniendo en cuenta la complejidad de los organismos y que estos se entienden cada vez más como sistemas en desarrollo que van modulando la expresión de sus genes continuamente, a partir de las señales internas y externas que reciben (CLIMENT et al., 2008).

En el ejemplo anterior sobre la resistencia al viento, la multiplicidad de variables ambientales mencionadas actúan sobre fenotipos complejos, en los que al menos la esbeltez del tronco, la arquitectura de la copa y las características de la raíz (anclaje) influirán en su resistencia, y estas a su vez, han sido moduladas a lo largo de la vida del árbol por su exposición al viento y los procesos de aclimatación (TAMASI et al., 2005, CouTAND et $a l .$, 2008). El mismo genotipo creciendo en otro suelo y otro clima, o en un rodal con una densidad distinta, o durante unos años en los que el régimen de viento haya sido diferente (por ejemplo), no habría desarrollado la misma esbeltez, ni la misma anchura de copa o disposición de ramas, ni la misma estructura de raíces.

\section{Del Genotipo al Fenotipo}

A pesar de los indudables avances en herramientas genéticas e informáticas, muchas veces la única relación que se puede establecer entre una potente colección de datos genómicos y los fenotípicos o fenómicos es estadística, a falta de un buen conocimiento las redes de procesos biológicos subyacentes y su organización. Un mapa genotipofenotipo de un carácter complejo sólo puede trazarse a través de la disección del carácter y la especificación de los procesos que ligan ambos niveles. Ello implica comprender a fondo los sistemas biológicos: sus componentes, propiedades, relaciones entre ellos y funciones. TAHRI-DAIZADEH et $a l$. (2003) señalan que si los caracteres complejos son la expresión de complejos sistemas biológicos, no puede esperarse que los genes y los caracteres estén relacionados por cadenas simples de eventos, sino por redes de relaciones.
Conocer bien el sistema biológico puede ayudar a definir bien el fenotipo asociado al sistema y aumentar la eficiencia de medición, ya que orienta sobre cuáles son las medidas más significativas en un panorama de fenotipos multidimensionales y caracteres complejos. Por otra parte, permitirá predecir con mayor precisión el fenotipo si se modifican las condiciones ambientales, es decir, permite modelizar el sistema de manera que puedan predecirse las consecuencias de los cambios en cualquiera de sus elementos, lo que amplía las posibilidades de manejo.

Empiezan a publicarse este tipo de modelos o mapas genotipo-fenotipo cada vez más completos, según avanza el conocimiento de todos los niveles de organización de las plantas y las señales y canales de comunicación entre ellos. Como ejemplos podemos citar el referido a la adaptación al frío (Howe et al., 2003), o la percepción del estrés mecánico y su respuesta en el crecimiento (TELEWSKI, 2006; Moulia et al., 2011).

Por otra parte, una visión más integral del fenotipo, que contemple varios caracteres simultáneamente y sus posibles correlaciones, puede incrementar la eficacia de la selección. La energía y recursos disponibles para realizar las funciones vitales (crecimiento, reproducción y mantenimiento) son limitados, por lo que cualquier inversión en una de ellas supone una reducción de los recursos disponibles para las demás. La distribución de estos recursos por tanto puede imponer compromisos que impidan la optimización simultánea de las funciones vitales (KoRICHEVA, 2002). Las limitaciones ambientales, como la exposición al viento o la sequía, pueden modular estos compromisos (CLIMENT et al., 2008). Dentro de los PMG, la selección sesgada hacia una de esas funciones puede comprometer la optimización simultánea de las demás.

En este sentido, el estudio del crecimiento de los árboles en el ámbito forestal se basa generalmente en su altura y diámetro, o incluso en el reparto de biomasa entre tronco y ramas, olvidando la cara oculta, es decir, la raíz, sobre todo en estudios de campo. Sin embargo el árbol es un todo integrado y la raíz tiene un papel fundamental en las funciones de crecimiento y mantenimiento, compartiendo la energía y los recursos con el resto de la planta y jugando un papel central en su adquisición. 
Existe variabilidad en la estrategia de colonización del suelo. Por ejemplo, al germinar algunas especies (quercíneas), la raíz es lo primero que comienza a desarrollarse, pudiendo alcanzar una longitud considerable antes de que el tallo comience a crecer, mientras que en otras (coníferas), el desarrollo de ambas partes suele ser más parejo. Las raíces tienen un periodo de crecimiento más extendido que el tallo a lo largo del año en muchas especies (HARRIs et al., 1995). Por otra parte, las raíces son órganos sumamente plásticos que se adaptan a las muy variables condiciones que encuentran en el suelo y reaccionan rápidamente ante estímulos que recibe la planta. Así, estímulos tan breves como flexiones de 20 segundos en una raíz de Arabidopsis, estimulan la formación de nuevas raíces en la parte convexa de la raíz flexionada en el $40 \%$ de las plantas ensayadas (RICHTER et al., 2009). En $P$. pinaster, la simulación de herbivoría mediante la aplicación de metil-jasmonato, provoca en dos semanas un incremento notorio de las raíces finas, mientras que se reduce el crecimiento de la parte aérea y de las raíces gruesas (MOREIRA et al., 2012). Las raíces acusan también la acción del viento y esto repercute en el reparto de biomasa aérea/radical, pudiendo existir una variabilidad genética en la sensibilidad y en el reparto. Estos ejemplos sobre la variabilidad genética, plasticidad y reactividad de la raíz ilustran que la integración fenotípica del crecimiento aéreo-radical permitiría una interpretación del valor genético de los individuos de una población de mejora diferente de la basada en la estimación de alturas y diámetros.

Sin duda, la comparación entre genotipos y fenotipos o genomas y fenomas de individuos, poblaciones o especies, puede aportar mucha información relevante para definir y caracterizar los sistemas biológicos. Existen ya muchos ensayos genéticos (de procedencias, progenies y clones) de variadas especies y en lugares diferentes, que ofrecen una fuente de valiosísima información en este sentido.

\section{EL VIENTO Y LA ESTABILIDAD MECÁNICA, DOS GRANDES OLVIDADOS}

\section{EI viento}

El efecto del viento en los árboles es conocido desde antiguo. Altera su morfología, anatomía y propiedades biomecánicas (TELEWSKI, 2006). Estas alteraciones se han denominado con el término genérico de tigmomorfogénesis (JAFFE, 1973). No obstante, el viento raramente se ha considerado hasta ahora en el análisis del comportamiento de las plantas, en particular a la hora de evaluar ensayos genéticos de especies forestales.

Recientemente se ha despertado en la comunidad científica internacional un interés mayor por el viento, como consecuencia de las grandes pérdidas en vidas humanas e infraestructuras ocasionadas por los huracanes (como Gudrun en 2005, Kyrill en 2007 y Klaus en 2009), sus devastadores efectos sobre los bosques y las predicciones de aumento de la intensidad de los vendavales en Europa. Los huracanes más fuertes que han afectado a Europa, como el de 1999, han producido un total de daños en árboles derribados equivalente, grosso modo, a la mitad de toda la madera cortada en Europa (excluida Rusia). En algunos países han supuesto más de 3 veces la posibilidad anual, y a escala de distrito forestal pueden haber representado más de 20 años de corta (GARDINER et al., 2010).

En el contexto del cambio climático en Europa, está previsto que los huracanes aumenten no su frecuencia pero sí su intensidad, de forma que se espera que los daños en pérdidas de volumen de madera provocadas por viento se multipliquen entre 2 y 4 veces para final de siglo (GARDINER et al., 2010). Pero además, el régimen ordinario del viento, con velocidades dentro del rango en el que no se esperan daños (menores de $60-70 \mathrm{~km} \cdot \mathrm{h}^{-1}$ ), tiene efectos notables sobre el crecimiento, la arquitectura y la rectitud de los troncos, que lo sitúan como una variable ambiental explicativa de primer orden. Por ejemplo, WATT et al. (2010) encuentran que la velocidad media anual del viento es el tercer factor en importancia, tras la temperatura y el agua disponible para las raíces, para definir el Índice de sitio en Pinus radiata. Estos efectos no catastróficos del viento, y en particular el efecto mecánico del viento sobre el crecimiento y la forma del árbol individual, merecen una atención especial, ya que en las respuestas de las plantas puede existir una componente genética importante.

El viento somete a los árboles a esfuerzos mecánicos, principalmente un balanceo que induce esfuerzos de tensión y compresión alternados, 
combinados con efectos de torsión sobre el tronco, ramas y raíces (QUIRK et al., 1975, QUIRK \& Freese, 1976a,b; NiKlas, 1999; De LANGRe, 2006; TelewsKI, 2006; Coder, 2010). El crecimiento de las plantas es extremadamente sensible al régimen de cargas crónico a que le somete el viento (Moulia et al., 2011). En un experimento con Pinus contorta, tras eliminar durante 6 años las flexiones inducidas por el viento mediante un sistema de tensores, se observó un aumento del $40 \%$ en crecimiento en altura y una reducción del $23 \%$ en crecimiento diametral (MENG et al., 2006). El mismo efecto mecánico que la oscilación por viento produce la vibración artificial de Pinus pinea para el aprovechamiento mecánico del piñón: MARTíneZ ZuRIMENDI et al. (2006) observaron una reducción significativa del 32\% en la elongación anual de los brotes en pinos vibrados frente a los no vibrados. En chopos, COUTAND et al. (2009) mostraron que tras una flexión momentánea del tronco, el crecimiento diametral primero cesaba aproximadamente durante 4 horas, y posteriormente se reiniciaba, alcanzando mayores valores de crecimiento que antes de la flexión durante 4-5 días. Además, se ha sugerido que la capacidad de adaptación al estrés mecánico está controlada genéticamente. Analizando 6 especies de árboles, CoUTAND et al. (2010) encontraron diferencias significativas en el umbral de estímulo mecánico (deformación por flexión) que modificaba el crecimiento secundario.

El estudio de los efectos tígmicos que los ambientes ventosos inducen en los troncos de los árboles, ha revelado significativos efectos en la anatomía de la madera, como el aumento del ángulo de las microfibrillas, de la densidad de la madera y de la lignificación, un incremento de la división celular en la dirección de la flexión, disminución de módulo de elasticidad (E) a la vez que aumento de la resistencia a la ruptura, o la aparición de canales resiníferos traumáticos. En la morfología del árbol se observa una menor relación H:D (troncos más bajos y más gruesos), hojas más pequeñas, entrenudos y ramas más cortas, uniones más gruesas de las ramas al tronco, así como alteración del porte, ya sea por rotura y pérdida de ramas o por la orientación de las ramas en respuesta a una dirección predominante del viento, reduciendo así la resistencia opuesta por el árbol (TELEWSKI, 2012; MoULIA et al., 2011).
Las raíces también son sensibles al viento y se aclimatan a los diferentes tipos de esfuerzo que el viento les impone (tracción, compresión y torsión), por ejemplo modificando el tamaño y forma de su sección transversal aumentando su resistencia (NiColl, 1996; StoKes \& MatTHECK, 1996; NIKLAS, 1999). También se ha sugerido la aparición de madera de compresión en las raíces (FAYLE, 1976), pero hay resultados contradictorios y en todo caso no es fácil identificarla en raíces.

El reparto de biomasa dentro del árbol se ve afectado por el viento, aunque el estímulo mecánico no afecta a la biomasa total producida, sino a su distribución: por una parte disminuye la biomasa aérea y aumenta la radical, y, dentro de la parte aérea, disminuye el crecimiento primario mientras que el secundario aumenta relativamente (CouTAND et al., 2008). El fenómeno se ha interpretado según el principio del equilibrio funcional (MÄKELÄ, 1999), que predice que en situación de estrés, la biomasa se dirige preferentemente a las partes de la planta donde es más eficiente para reducir el estrés.

Estos cambios anatómicos y morfológicos modifican el reparto de los recursos disponibles para realizar las funciones vitales y por tanto su papel puede ser muy relevante sobre caracteres de interés como el volumen de madera y su calidad.

El viento por tanto tiene un notable efecto sobre el crecimiento (disminuye la proporción biomasa aérea/radical) y la forma de los troncos (aumenta la conicidad), haciéndose de esta manera más resistentes. Esta es una importante modulación de la forma para mantenerse en pie en un ambiente de esfuerzos variables. Es bien conocida por los forestales la trascendencia de la densidad de plantación en la esbeltez o de la apertura de claros en la masa en el riesgo de derribo por el viento. A pesar de ello, muchos de los modelos de crecimiento existentes no contemplan la tigmomorfogénesis y no recogen por tanto los efectos de aclimatación al viento (Moulia et al., 2006), ni tampoco se tiene en cuenta la capacidad de adaptación al viento en la selección en los PMG.

\section{La estabilidad mecánica}

Hace unos 400 millones de años, "la invasión botánica de la tierra fue realmente una invasión 
del aire" (NIKLAS, 1997). Desde entonces, los árboles han evolucionado como seres de larga vida y gran tamaño, con una estructura muy esbelta, que se enfrentan al importante reto mecánico de mantenerse erguidos en un ambiente de esfuerzos variables. Durante su desarrollo, deben ir ajustando sus características (geometría y constitución material) para evitar fallos mecánicos (roturas, caídas, etc.) a la vez que compiten por colonizar el espacio para abastecerse de agua, luz y nutrientes. La estabilidad mecánica ha tenido que evolucionar en interacción con el resto de los caracteres vitales, pero no ha recibido, hasta ahora, gran atención por parte de los mejoradores.

Mantenerse en pie en un ambiente sometido a esfuerzos mecánicos variables no es una función trivial para un ser con la base fija, mucha altura, gran esbeltez y una copa más o menos amplia. Actúan sobre el árbol cargas debidas a cambios en la presión atmosférica (que originan viento), cargas debidas a la gravedad (nieve, hielo, el propio peso, incluyendo la presencia temporal de frutos y hojas, etc.) y estímulos de contacto como el golpeteo de la lluvia o el granizo, o provocados por las plantas contiguas, animales, etc.

Para un árbol, las condiciones ambientales cambian con rapidez. Sin embargo, la fuerza gravitatoria es un factor estable, que afecta a todos los organismos y ha contribuido a guiar su evolución (NiKLAS, 1998; VOLKMANN \& BALUSKA, 2006). Merced a esta evolución, los árboles consiguen mantenerse en pie en las distintas fases de su desarrollo, mediante mecanismos activos de control postural y desarrollando un buen sistema de anclaje (raíces). Estos mecanismos tienen repercusiones en la proporción de biomasa aérea/radical, el crecimiento en altura y diámetro, la conicidad y excentricidad del tronco, las características mecánicas de la madera, su calidad y la rectitud del fuste, entre otros. La rectitud del fuste es un carácter con importancia industrial, pero también biológica si se toma como punto de partida la estabilidad mecánica del árbol. Nos extenderemos a continuación en este aspecto.

\section{La rectitud en la mejora: perspectiva biomecánica}

La forma del tronco es una de las características que junto con el crecimiento y la resistencia a enfermedades y plagas es definida como carácter objetivo en la mayoría de los PMG de árboles para la obtención de madera de calidad (MulLin et al., 2011). Sin embargo, la forma ha sido definida generalmente de una manera imprecisa y evaluada con métodos subjetivos, lo que ha generado unos resultados muy dispares a la hora de estimar el grado de contribución genética (heredabilidad) en distintos PMG (Cornelius, 1994; Sierra DE GRAdo et al., 1997). La definición de la "forma" del tronco varía de unos autores a otros: puede significar rectitud, verticalidad, conicidad, englobar o no bifurcaciones, bayonetas, conicidad, ramosidad, etc. En esta ocasión nos referiremos a la rectitud, entendiendo que un tronco será más o menos recto según las curvaturas que presente, si bien muchas veces la aparición de curvaturas en el tronco se debe a una inclinación inicial, como veremos más adelante. La rectitud puede cambiar a lo largo de la vida del árbol, en función de factores ambientales a veces erráticos (vendavales, nevadas fuertes, apertura de claros en la masa que inducen crecimiento fototrópico, etc.). Estos cambios son en gran medida aleatorios y pueden repercutir en bajas heredabilidades y débiles correlaciones juvenil-adulto.

Las plantas detectan su posición relativa en el campo gravitatorio a través de mecanismos graviperceptivos muy sensibles y ajustan su forma y posición mediante procesos motores activos para mantenerse en pie (Moulia \& FOURNIER, 2009). Cuando el viento u otra causa genera una desviación del tallo suficientemente grande, este tenderá a reorientarse para recuperar la posición vertical (regulación gravitrópica), lo que muchas veces conlleva la formación de curvaturas en el tallo y la producción de madera de reacción. Por tanto, la rectitud de un tronco de árbol no sólo va a depender de la aparición de factores que desvían al árbol de su posición de equilibrio, sino de la propia capacidad del árbol de enderezarse. El enderezamiento requiere también un tiempo para producirse y la rectitud del tronco irá variando con él progresivamente.

Esta perspectiva ofrece un planteamiento diferente para seleccionar árboles de buena rectitud: en lugar de evaluar la rectitud a una edad determinada, evaluar la capacidad del árbol de enderezarse y la eficiencia de ese proceso (enderezarse más en el mínimo tiempo y con el mínimo coste en tér- 
minos de madera de reacción producida). Un genotipo capaz de re-enderezarse de manera óptima ante cualquier eventualidad puede ser más interesante para el PMG que un árbol derecho porque jamás se ha visto forzado a torcerse.

\section{El enderezamiento}

El proceso de enderezamiento es complejo y puede estar sometido a muchos niveles de regulación genética. Se desencadena mediante el movimiento de estatolitos (orgánulos pesados como los amiloplastos) en el citoplasma celular, involucra el transporte lateral de auxina hacia el lado inferior del tallo inclinado y origina una expresión génica diferencial (BASTIEN et al., 2013).

Se conoce bien la cinemática del enderezamiento de plantas jóvenes de $P$. pinaster artificialmente inclinadas (LouP et al., 1991; FouRNIER et al., 1994; SIERRA DE GRADO et al., 2008). En él están implicadas reacciones gravitrópicas primarias y secundarias, es decir dependientes del crecimiento primario y secundario respectivamente. En ambos casos, la curvatura gravitrópica (curva el tallo hacia arriba para recuperar la verticalidad) es seguida por una contra-curvatura, llamada reacción autotrópica (contrarresta la curvatura gravitrópica tratando de enderezar el tallo en su posición vertical). El autotropismo es entendido como una curvatura de compensación, tanto en zonas de crecimiento primario (MESKAUSKAS et $a l ., 1999$; TARUI \& IINO, 1997; STANKOVICC et $a l$., 1998) como con crecimiento secundario (COUTAND et al., 2006). Este patrón es generalizable a otras especies (BASTIEN et al., 2013).

Dos procesos motores están implicados en estos movimientos: uno, la curvatura del segmento apical a través del crecimiento primario, producida por una diferente elongación celular en la cara superior e inferior del tallo en la base del ápice (Tomos et al., 1989), y dos, una curvatura del segmento basal con crecimiento secundario, causada por la producción de madera de reacción en la cara inferior del tallo y que generalmente va acompañada de mayor crecimiento radial en dicha cara inferior (FOURNIER et al., 1994; MOULIA \& FourNIER, 2009).

La biomecánica de esta curvatura basal promovida por el crecimiento secundario ha sido estudiada en detalle. La pared celular secundaria experimenta contracción o expansión longitudi- nal durante su diferenciación ("esfuerzos de maduración"). En el caso de las coníferas, si un tallo está inclinado se producen dos tipos de madera (TIMMEL, 1986): una madera con anatomía celular estándar, llamada "madera normal", en la que la pared celular experimenta contracción longitudinal al diferenciarse y se produce en la cara superior del tallo, y una madera de reacción con células más redondeadas y paredes celulares más gruesas y más lignificadas, denominada "madera de compresión", que presenta expansión longitudinal en la diferenciación celular y se presenta en la parte inferior del tallo. Estas diferentes tensiones de maduración generan tensiones mecánicas en el tronco. Cuando un sector de madera de compresión se produce en el lado inferior de un tallo inclinado, la asimetría de tensiones de maduración produce que el tallo se curve hacia arriba. En frondosas se produce otro tipo de madera de reacción, la madera de tensión, en la cara superior del tallo, que también produce una curvatura hacia arriba. AlmÉrAs et al. (2005) encontraron que el principal factor de la reorientación del tronco en 17 especies de árboles era la asimetría en las tensiones de maduración $(\alpha)$, que explicaba, por sí sola, un $57 \%$ de las reorientaciones.

Tanto la duración del proceso antes de llegar a recuperar totalmente la rectitud, como la cantidad de madera de reacción producida durante el mismo dependen del tamaño del fuste y de la eficiencia de la madera de reacción producida y tienen también un componente genético (FourNIER et $a l$., 2006; SIERRA DE GRADO et $a l$., 2008; Moulia \& Fournier, 2009). Se ha demostrado que en $P$. pinaster existe una variabilidad geográfica en $\alpha$ (MENÉNDEZ, 2010) y se ha sugerido que las procedencias de $P$. pinaster con buena rectitud presentan unas reacciones gravitrópicas y autotrópicas secundarias mayores que las otras procedencias, que se corresponden con valores mayores de $\alpha$ en las procedencias con buena rectitud (SIERRA DE GRADO et al., 2008). Además la heredabilidad de las variables biomecánicas relacionadas es elevada (CALLEJA, 2011).

También se ha encontrado variabilidad genética inter e intraespecífica en otras variables relacionadas con el gravitropismo y el enderezamiento, como en el tiempo de presentación 
(tiempo necesario para percibir el estímulo), en la expresión de genes implicados (CouTAND et al., 2010; RAMOS et al., 2012), en la rapidez de la respuesta gravitrópica primaria y secundaria, así como en la cantidad de madera de reacción formada (BA et $a l ., 2010$ ), en la eficiencia de la respuesta secundaria y en las características anatómicas asociadas (ALMÉRAS et al., 2009) y de la madera de compresión (SHELbourne et al., 1969), etc. El proceso de curvatura debido a la formación de madera de reacción se ha cuantificado en modelos biomecánicos (FouRNIER et al., 1994; FoURCAUD et al., 2003; FoURCAUD \& LAC, 2003; AlmÉrAs et al., 2009; BASTIEN et al., 2013), que suponen una herramienta muy útil a la hora de estudiar la importancia relativa de las variables implicadas en la variabilidad (y por tanto más relevantes de medir).

Hay mucho todavía por explorar. La capacidad de enderezamiento puede estar relacionada, como hemos visto, con la rectitud. La eficiencia de la corrección gravitrópica también se ha relacionado con la capacidad de capturar la luz (AlmÉras et $a l ., 2009$ ) y con la adaptación a la sequía (BA et $a l ., 2010)$. El desarrollo de madera de reacción o un mayor crecimiento relativo en diámetro contribuyen a enderezar el tallo, pero a la vez pueden detraer recursos para el crecimiento en altura, lo que repercute en la capacidad de competir por la luz. Estas compensaciones podrían estar relacionadas con la correlación genética negativa que se ha encontrado en ocasiones entre el crecimiento en altura o volumen y la rectitud (Aguiar et al., 2003; Dieters, 1996). Así mismo, la producción de madera de compresión permite la reorientación del tallo pero podría reducir la capacidad de transporte de agua (BA et al., 2010), lo que en un ambiente xérico impondría fuertes compromisos.

\section{CONCLUSIÓN}

La visión integrada de las características fenotípicas de un árbol y la comprensión exhaustiva de los sistemas biológicos que regulan los caracteres de interés puede orientar la selección dentro de los PMG en un nuevo y más eficiente rumbo. En un contexto de cambio climático esta vía es, si cabe, más importante aún.
El viento como factor ambiental y la estabilidad mecánica como necesidad del árbol juegan un papel relevante en el espacio multidimensional donde se definen los fenotipos y tienen una gran repercusión en caracteres de interés como el volumen del tronco, la rectitud y la calidad de la madera. Los procesos biomecánicos de enderezamiento del tallo ayudan a la planta a mantener su estabilidad mecánica y están relacionados directamente con la rectitud del tronco, con una variabilidad genética asociada a diferentes niveles. Sin embargo, hasta ahora la importancia de estos aspectos no ha sido suficientemente reconocida.

El conocimiento de los sistemas biológicos que regulan la aclimatación y adaptación al viento, y de los procesos biomecánicos implicados en la estabilidad mecánica del árbol, desde los genes y su expresión, hasta su interrelación con otras funciones vitales y variables ambientales, permitirá entender muchos aspectos del funcionamiento de los árboles, así como mejorar los modelos que posibilitan predecir su comportamiento.

\section{BIBLIOGRAFÍA}

Aguiar, A.; Almeida, M.H. \& Borralho, N.; 2003. Genetic control of growth, wood density and stem characteristics of Pinus pinaster in Portugal. Silva Lusitana 11(2): 131-139.

Alméras T.; ThibAut A. \& GRIL J.; 2005 Effect of circumferential heterogeneity of wood maturation strain, modulus of elasticity and radial growth on the regulation of stem orientation in trees. Trees-Structure and function 19(4): 457-467

Alméras, T.; Derycke M.; JaOUen, G.; BEAUChÊNE, J. \& Fournier, M.; 2009. Functional diversity in gravitropic reaction among tropical seedlings in relation to ecological and developmental traits. J. Exp. Bot. 60: 4397-4410

BA, E.H.M.; SAlin, F.; FourCAUd, T. \& STOKES, A.; 2010 Reorientation strategies in leaning stems of young maritime pine (Pinus pinaster) and loblolly pine (Pinus taeda). Int. Assoc. Wood Anat. J. 31: 465-480.

Bastien, R.; Bohr, T.; Moulia, B. \& Douady, S. 2013. Unifying model of shoot gravitropism reveals proprioception as a central fea- 
ture of posture control in plants. Proceedings of the National Academy of Sciences 110(2): 755-760

Busov, V.B.; Brunner, A.M. \& Strauss, S.H.; 2008. Genes for control of plant stature and form. New Phytologist 177(3): 589-607.

Calleja, A.; 2011. Variabilidad de la capacidad de enderezamiento entre progenies de Pinus pinaster Ait. Tesis de máster. Universidad de Valladolid.

Chambel, R.; Climent, J.; Alía, R. \& VALLADARES, F.; 2005 Phenotypic plasticity: a useful ramework for understanding adaptation in forest species Inv. Agrar: Sist. Rec. For. 14(3): 334-344.

Climent, J.; Prada, M.A.; Calama, R.; Chambel, M.R.; De Ron, D.S., \& Alía, R.; 2008. To grow or to seed: ecotypic variation in reproductive allocation and cone production by young female Aleppo pine (Pinus halepensis, Pinaceae). Am. J. Bot. 95(7): 833-842.

Coder, K.D.; 2010. Root Strength and Tree Anchorage. University of Georgia. Warnell School of Forestry \& Natural Resources monograph publication WSFNR10-19*.Pp.88

CoRNELIUS, J.; 1994 Heritabilities and additive genetic coefficients of variation in forest trees. Can. J. For. Res. 24(2): 372-379, 10.1139/x94-050.

COST 4131/12; 2012. Memorandum of understanding for the implementation of a European Concerted Research Action designated as COST Action FP1202 Strengthening conservation: a key issue for adaptation of marginal/peripheral populations of forest tree to climate change in Europe (map-fgr) 2012 www.parlament.gv.at/.../imfname_10035606.pdf?

Coutand, C.; Martin, L.; LeblanC-Fournier, N.; Decourteix, M.; Julien, J \& Moulia, B.; 2009. Strain mechanosensing quantitatively controls diameter growth and PtaZFP2 gene expression in poplar. Plant Physiology 151: 223-232.

Coutand, C.; Dupraz, C.; Jaouen, G.; Ploquin, S., \& ADAM, B.; 2008. Mechanical stimuli regulate the allocation of biomass in trees: demonstration with young Prunus avium trees. Ann. Bot. 101(9): 1421-1432.

Coutand, C.; Chevolot, M.; Rowe, N.; Lacointe, A. \& Scotti, I.; 2010
Mechanosensing of stem bending and its interspecific variability in five neotropical rainforest species. Ann. Bot. 105: 341-347.

DiETERS, M.J.J.; 1996. Genetic parameters for slash pine (Pinus elliottii) grown in southeast Queensland, Australia: Growth, stem straightness and crown defects Forest Genetics 3(1): 27-36.

Ellis, B.; Jansson, S.; Strauss, S.H. \& Tuskan, G.A.; 2010. Why and how Populus became a "model tree". En: S. Jansson, R. Bhalerao \& A. Groover (eds), Genetics and Genomics of Populus: 3-14. Springer.

FAYLE, D.C.F.; 1976. Notes: stem sway affects ring width and compression wood formation in exposed root bases. For. Sci. 22(2): 193-194.

FourCAUD, T. \& LAC, P.; 2003. Numerical modelling of shape regulation and growthstresses in trees I. An incremental static ?nite element formulation. Trees 17: 23-30.

Fourcaud, T.; Blaise, F.; Lac, P.; Castera, P. \& De RefFye, P., 2003. Numerical modeling of shape regulation and growth stresses in trees II. Implementation in the AMAPpara software and simulation of tree growth. Trees 17: 31-39.

Fournier, M.; BaIllerres, H. \& Chanson, B.; 1994. Tree Biomechanics: Growth, cumulative prestresses and reorientations. Biomimetics 2(3): 229-252

Fournier, M.; Stokes, A.; Coutand, C.; Fourcaud, T. \& Moulia, B.; 2006. Tree biomechanics and growth strategies in the context of forest functional ecology. In: A. Herrel, T. Speck \& N.P. Rowe (eds.), Ecology and biomechanics: 1-33. Taylor \& Francis CRC Press.

Freimer, N. \& SABATTI, C.; 2003 The human phenome project. Nat. Genet. 34:15-21.

GARDINER, S.E. et al.; 2010. DG Environment entitled "Destructive Storms in European Forests: Past and Forthcoming Impacts". This report is available online at http://ec.europa.eu/environment/forests/fpro tection.htm

Gaspar, M.J.; De-Lucas, A.I.; Alía, R.; Paiva, J.A.P.; Hidalgo, E.; LouZADA, J.; AlmEIDA, H. \& GonZÁlez-MarTínEZ, S.C.; 2009. Use of molecular markers for estimating breeding parameters: a case study in a Pinus 
pinaster Ait. progeny trial. Tree Genetics \& Genomes 5: 609-616.

GonZÁleZ-MARTíneZ, S.C.; WhEELER, N.C.; Ersoz, E.; Nelson, C.D. \& Neale, D.B.; 2007. Association genetics in Pinus taeda L. I. Wood property traits. Genetics 175 : 399-409

HARRIS, J.R.; BASSUK, N.L.; ZoBEL, R.W.; WhitLow, T.H.; 1995. Root and shoot growth periodicity of Green Ash, Scarlet Oak, Turkish Hazelnut, and Tree Lilac. J. Am. Soc. Hort. Sci. 120(2): 211-216.

Howe, G.T.; AitKen, S.N.; Neale, D.B.; JeRMSTAD, K.D.; WheELER, N.C. \& CHEN, T.H.; 2003. From genotype to phenotype: unraveling the complexities of cold adaptation in forest trees. Can. J. Bot. 81(12): 1247-1266.

JAFFE, M.J.; 1973. Thigmomorphogenesis: the response of plant growth and development to mechanical stimulation. Planta 114: 143-157.

JANSSON, S.; BhaleraO, R. \& GroOver, A. (Eds) 2010. Genetics and Genomics of Populus. Springer. Heidelberg.

Koricheva, J.; 2002. Meta-analysis of sources of variation in fitness costs of plant antiherbivore defenses. Ecology 83: 176-190.

De LANGRe, E.; 2008. Effects of wind on plants. Annu. Rev. Fluid Mech. 40:141-168

Loup, C.; Fournier, M.; Chanson, B. \& Moulia, B.; 1991. Redressements, contraintes de croissance et bois de réaction dans le bois d'un jeune Pinus pinaster Ait. artificiellement incliné. In: B. Thibaut (ed), Proceedings of the third Seminar "Architecture, Structure, Mécanique de l'Arbre" Montpellier LMGC Université Montpellier II. Montpellier.

MÄKELÄ, A.; 1999. Acclimation in dynamic models based on structural relationships. Functional Ecology 13: 145-156

Martínez Zurimendi, P.; Álvarez, J.M.; PANDO FERnÁndeZ, V.; DomíngueZ, M.; Gordo, J.; FinAT, L. \& SiERRA DE GRAdo, R.; 2009. Efectos del vibrado del pino piñonero (" Pinus pinea" L.) en el vigor de los árboles: densidad de copa, crecimiento de guías y parásitos de debilidad. Inv. Agrar.; Sist. Rec. For. 18(1): 50-63.

Mátyás, C.; Vendramin, G.G., \& Fady, B.; (2009). Forests at the limit: evolutionary-genetic consequences of environmental changes at the receding (xeric) edge of distribution. Report from a research workshop. Ann. For. Sci. 66(8).

MÁTYÁs, C.; 2010. Forecast needed for retreating forest. Nature 646.

MENÉNDEZ, M.; 2010. Variabilidad entre procedencias de la capacidad de enderezamiento de Pinus pinaster Ait. Tesis de máster. Universidad de Valladolid. Palencia.

Meng, S. X.; LiefFers, V.J.; ReID, D.E.; RUDNICKI, M.; SILINS, U. \& JIN, M.; 2006. Reducing stem bending increases the height growth of tall pines. J. Exp. Bot. 57(12): 3175-3182.

Meskauskas, A.; Jurkoniene, S. \& Moore, D.; 1999. Spatial organization of the gravitropic response in plants: applicability of the revised local curvature distribution model to Triticum aestivum coleoptiles. New Phytologist 143(2): 401-407.

MoreIrA, X.; ZAS, R. \& SAMPEdRO, L.; 2012. Genetic variation and phenotypic plasticity of nutrient re allocation and increased fine root production as putative tolerance mechanisms inducible by methyl jasmonate in pine trees. J. Ecol. 100(3): 810-820.

Moulia, B.; Der loughian, C.; Bastien, R.; Martin, L.; Rodriguez, M.; GoURCILlEAU, D.; Barbacci, A.; BADEl, E.; Franchel, J.; Lenne, C.; Roeckel-Drevet, P.; Allain, J.M.; Frachisse ， J.M.; De Langre, E.; Coutand, C.; Fournier-Leblanc, N. \& JULIEN,J.L.; 2011. Integrative mechanobiology of growth and architectural development in changing mechanical environments. In: P. Wojtaszek (ed.), Mechanical Integration of Plant Cells and Plants: 269302. Springer, Series: Signaling and Communication in Plants, Springer-Verlag $\mathrm{GmbH}$. Berlin. Heidelberg.

Moulia, B. \& Fournier, M.; 2009 The power and control of gravitropic movements in plants: a biomechanical and systems biology view. J. Exp. Bot. 60(2):461-86

Moulia, B.; Coutand, C. \& Lenne, C.; 2006 Posture control and skeletal mechanical acclimation in terrestrial plants: implications for mechanical modelling of plant architecture. Am. J. Bot. On press (2006-108R).

Mullin, T.J.; ANDERSSON, B.; BAstien, J.-C.; Beaulieu, J.; Burdon, R.D.; DVORAK, W.S.; 
KInG, J.N.; Kondo, T.; KRAKOWSKI, J.; LEE, S.J.; MCKeAND, S.E.; PÂQUES, L.; RAFFIN, A.; Russell, J.; SkrøPPA, T.; StOehr, M. \& YANCHUK, A.D.; 2011. Chapter 2: Economic importance, breeding objectives and achievements. In: C. Plomion, J. Bousquet \& C. Kole (eds.), Genetics, Genomics and Breeding of Conifers: 40-127. Science Publishers, Inc. Edenbridge Ltd. Enfield, NH.

NiCOLL, B.C. \& RAY, D.; 1996. Adaptive growth of tree root systems in response to wind action and site conditions. Tree Physiology 16: 891-898.

NIKLAS, K.J.; 1997. The Evolutionary Biology of Plants. University of Chicago Press. Chicago.

NiKLAS, K.J.; 1998. Effects of vibration on mechanical properties and biomass allocation pattern of Capsella bursa-pastoris (Cruciferae). Ann. Bot. 82: 147-156.

NiKLAS, K.J.; 1999. Variations of the mechanical properties of Acer saccharum roots. J. Exp. Bot. 50(331): 193-200.

OraguZIE, N.C.; RIKKERINK, E.H.; GARDINER, S. E., \& SILVA, H.D.; 2007. Association mapping in plants. Springer-Verlag GmbH. Berlin.

QuirK, J.T.; SMith, D.M., \& FreESE, F.; 1975. Effect of mechanical stress on growth and anatomical structure of red pine (Pinus resinosa Ait.): torque stress. Can. J. For. Res. 5(4): 691-699.

QUIRK, J.T. \& FREESE, F.; 1976a. Effect of mechanical stress on growth and anatomical structure of red pine: compression stress. Can. J. For. Res. 6(2): 195-202.

QUIRK, J.T. \& FREESE, F.; 1976b. Effect of mechanical stress on growth and anatomical structure of red pine: stem vibration. Can. J. For. Res. 6(3): 375-381.

Ramos, P.; Le Provost, G.; Gantz, C.; Plomion, C. \& HerrerA, R.; 2012, Transcriptional analysis of differentially expressed genes in response to stem inclination in young seedlings of pine. Plant Biology 14: 923-933

RESENDE, M.D.; RESENDE, M.F. JR; SANSALONI, C.P.; Petroli, C.D.; Missiaggia, A.A.; Aguiar, A.M.; ABAD, J.M.; TAKAHASHI, E.K.; RoSADO, A.M.; FARIA, D.A.; PAPPAS, G.J. JR; Kilian, A. \& Grattapaglia, D.; 2012. Genomic selection for growth and wood quality in Eucalyptus: capturing the missing heritability and accelerating breeding for complex traits in forest trees. New Phytologist, 194(1): 116-28

ReSENDE, M.F. JR; MuÑOZ, P.; Acosta, J.J.; Peter, G.F.; DAVIS, J.M.; 2012. Accelerating the domestication of trees using genomic selection: accuracy of prediction models across ages and environments. New Phytologist 193(3): 617-24

Richter, G.L.; Monshausen, G.B.; Krol, A. \& GILROY, S.; 2009. Mechanical stimuli modulate lateral root organogenesis. Plant physiology 151(4): 1855-1866.

Shelbourne, C.J.A.; Zobel, B.J. \& StoneCYPHER, R.W.; 1969. The inheritance of compression wood and its genetic and phenotypic correlations with six others traits in five-yearold Loblolly pine. Silvae Genet. 18: 43-47

Sierra-DE-Grado, R.; Moulia, B.; Fournier, M.; AlíA, R. \& Díez-Barra, R.; 1997. Genetic control of stem form in Pinus pinaster Ait. seedlings exposed to lateral light. Trees, Structure and Function 11: 455-461.

Sierra-De-Grado, R.; PANdo, V.; Martinez Zurimendi, P.; Bascones, E.; Peñalvo, A. \& Moulia, B.; 2008. Biomechanical differences in the stem straightening process among Pinus pinaster provenances. A new approach for early selection of stem straightness. Tree Physiology 28: 835-846.

Somers, D.; LANGridge, P. \& Gustafson, P.J. (eds.); 2009. Plant genomics: methods and protocols. The Humana Press, Totowa

Stankovic, B. \& Volkmann, D.; 1998. Autotropism, automorphogenesis and gravity. Physiologia Plantarum 102: 328-335.

StOKES, A. \& MATTHECK, C.; 1996. Variation of wood strength in tree roots. J. Exp. Bot. 47 (298): 693-699.

TAhri-Daizadeh, N.; Tregouet, D.A.; NicAud, V.; Manuel, N.; Cambien, F. \& Tiret, L.; 2003. Automated detection of informative combined effects in genetic association studies of complex traits. Genome Res. 13: 1952-1960

TAMASI, E.; STOKES, A.; LASSERRE, B.; DANJON, F.; Berthier, S.; Fourcaud, T. \& Chiatante, D.; 2005. Influence of wind loading on root system development and architecture in oak (Quercus robur L.) seedlings. Trees 19(4): 374-384. 
TARUI Y. \& IINO, M., 1997 Gravitropism of oat and wheat coleoptiles: dependence on the stimulation angle and involvement of autotropic straightening. Plant and Cell Physiology 38(12): 1346-1353.

TelewsKi, F.W. \& JAFFE, M.J.; 1986. Thigmomorphogenesis: the role of ethylene in the response of Pinus taeda and Abies fraseri to mechanical perturbation. Physiologia Plantarum 66(2): 227-233.

TelewsKI, F.W.; 1995. Wind induced physiological and developmental responses in trees. In: M. P. Coutts and J. Grace (eds.), Wind and trees: 237-263. Cambridge University Press. Cambridge.

Telewski, F.W.; 2006. A unified hypothesis of mechanoperception in plants. Am. J. Bot. 93(10): 1466-1476.

TELEWSKI, F.W.; 2012. Is windswept tree growth negative thigmotropism? Plant Science 184: 20-28.

Tomos, A.D.; Malone, M. \& Pritchard, J.; 1989. The biophysics of differential growth. Env. Exp. Bot. 29(1): 7-23
Timell, T.E.; 1986. Compression wood in gymnosperms. Springer-Verlag, Heidelberg, Germany.

Vasquez Gross, H.A.; Yu, J.J.; Figueroa, B.; Gessler, D.D.; Neale, D.B. \& Wegrzyn, J.L.; 2013. CartograTree: connecting tree genomes, phenotypes and environment. Molecular ecology resources.

VolKmanN, D. \& BALUSKA, F.; 2006. Gravity: one of the driving forces for evolution. Protoplasma 229: 143-148

Watt, M.S.; Palmer, D.J.; Kimberley, M.O.; Hock, B.K.; PAYN, T.W. \& LowE, D.J.; 2010. Development of models to predict Pinus radiata productivity throughout New Zealand. Can. J. For. Res. 40(3): 488-499.

WeAVer, J.E. \& Himmel, W.J.; 1929. Relation Between the Development Of Root System and Shoot Under Long- And Short-Day Illumination. Agronomy \& Horticulture Faculty Publications. Paper 503.

Zobel, B. Y TALBert, J.; 1984. Técnicas de mejoramiento genético de árboles forestales. Limusa. 